\title{
Situaciones que Originan Emociones en Estudiantes de Matemáticas
}

\section{Situações que Originam Emoções em Estudantes de Matemáticas}

\author{
María S. García-González * \\ ORCID iD 0000-0001-7088-1075 \\ Brenda Ramírez-Gómez ${ }^{* *}$ \\ ORCID iD 0000-0002-5917-0990 \\ Catalina Navarro-Sandoval ${ }^{* * *}$ \\ ORCID iD 0000-0001-5214-0062
}

\begin{abstract}
Resumen
A fin de ampliar la comprensión sobre las situaciones que originan emociones de estudiantes de bachillerato mexicano, se planteó una investigación a manera de replicación de los estudios realizados por Martínez-Sierra y García-González $(2014 ;$ 2017). Para tal efecto, se modificó solamente la modalidad de bachillerato, y se conservó el marco teórico-metodológico del estudio original, por lo que la teoría de la Estructura Cognitiva de las Emociones guio la recolección y el análisis de datos. Con base en los hallazgos, podemos decir que la modalidad del bachillerato no influye las emociones de los estudiantes, pues se encontraron emociones y situaciones desencadenantes parecidas al estudio original. Al parecer, las metas académicas que se plantea el estudiante son las que desencadenan sus emociones, si éstas se cumplen las emociones que se experimentan son positivas, de lo contrario son negativas, resultado que refuerza los hallazgos del estudio original. Las situaciones desencadenantes encontradas fueron: 1) entender los temas, 2) las preguntas y tareas en clase, 3) acreditar la materia, 4) resolver problemas, 5) comprender los temas, 6) el interés por las matemáticas y 7) la dificultad de las matemáticas, éstas originan ocho tipos de emociones, miedo, esperanza, júbilo, congoja, reproche, autorreproche, agrado y desagrado.
\end{abstract}

Palabras clave: Emociones de estudiantes. Matemáticas. Teoría de la estructura cognitiva de las emociones. Bachillerato. Estudio de replicación.

\section{Resumo}

Com o objetivo de ampliar a compreensão das situações que causam emoções em estudantes mexicanos do ensino médio, foi proposta uma investigação como uma réplica dos estudos realizados pelo Martínez-Sierra y GarcíaGonzález $(2014$; 2017). Para tanto, apenas a modalidade de escola foi modificada, e o referencial teóricometodológico do estudo original foi preservado, de forma que a teoria da Estrutura Cognitiva das Emoções norteasse a coleta e análise dos dados. Com base em descobertas, podemos afirmar que a modalidade de ensino médio não influencia as emoções dos alunos, uma vez que foram encontradas emoções e situações desencadeantes semelhantes ao estudo original. Aparentemente, os objetivos acadêmicos que o aluno estabelece são aqueles que

\footnotetext{
* Dra. en Matemática Educativa por el Centro de Investigación y Estudios Avanzados del Instituto Politécnico Nacional (Cinvestav, IPN). Profesora titular de la Facultad de Matemáticas de la Universidad Autónoma de Guerrero (UAGro), Chilpancingo, Guerrero, México. E-mail: mgargonza@ gmail.com.

** Licenciada en Matemática Educativa por la Universidad Autónoma de Guerrero (UAGro). Profesora interina de la Escuela Primaria Carmen Serdán, Cuernavaca, Morelos, México. E-mail: brenda26.ramgom@gmail.com.

**** Dra. en Matemática Educativa por la Universidad Autónoma de Guerrero (UAGro). Profesora titular de la Facultad de Matemáticas de la Universidad Autónoma de Guerrero (UAGro), Chilpancingo, Guerrero, México. Email: nasacamx@yahoo.com.mx.
} 
desencadeiam suas emoções, se estes forem atendidos as emoções vivenciadas são positivas, caso contrário, são negativas, resultado que reforça os achados do estudo original. As situações desencadeadoras encontradas foram: 1) compreensão dos tópicos, 2) as questões e tarefas em aula, 3) credenciamento da disciplina, 4) resolução de problemas, 5) compreensão dos tópicos, 6) interesse em matemática e 7) Dificuldade da matemática, das quais se originam oito tipos de emoções, medo, esperança, alegria, angústia, reprovação, autocensura, gosto e antipatia.

Palavras-chave: Emoções do aluno. Matemática. Teoria da estrutura cognitiva das emoções. Ensino médio. Estudo de replicação.

\section{Introducción}

Esta investigación se centra en el bachillerato (15-18 años), último nivel escolar obligatorio en México, en donde la importancia de las matemáticas se agudiza, al igual que los problemas de los estudiantes para acreditar los cursos que involucran el pensamiento matemático, dichos problemas son de naturaleza diversa, desde los cognitivos hasta los afectivos. Al respecto, desde la Educación Matemática diversas investigaciones contribuyen a aminorar estos problemas, con el fin de mejorar el aprendizaje de las matemáticas.

Nuestra investigación se une a este fin desde la perspectiva del denominado Dominio Afectivo, en particular desde el estudio de las emociones. El Dominio Afectivo es el campo de investigación referido al estudio de aspectos inherentes a lo humano, llamados constructos (actitudes, emociones, motivación, creencias, etcétera) que influyen y van formando la vida escolar y social de estudiantes y profesores (GARCÍA-GONZÁLEZ; MARTÍNEZ-SIERRA, 2018). Los estudios realizados en este campo han mostrado que el afecto tiene una alta influencia en el aprendizaje matemático escolar (DIEGO-MANTECÓN; CÓRDOBAGÓMEZ, 2019; IBARRA-GONZÁLEZ; ECCIUS-WELLMANN，2018; DI MARTINO; SABENA, 2011; MARTÍNEZ-PADRÓN, 2008; HANNULA, 2002; GÓMEZ-CHACÓN, 2000a).

El interés por el estudio de las emociones puede atribuirse a la importancia de la naturaleza emocional de los contextos educativos (SCHUTZ; PEKRUN, 2007). Por ello, cada vez más áreas se han involucrado en su estudio, entre ellas, la lengua (SHAO; PEKRUN; NICHOLSON, 2019), la ciencia (RETANA-ALVARADO et al., 2019) y las matemáticas (ROTH; WALSHAW, 2019). En el contexto propio de la Educación Matemática, los inicios de la investigación sobre emociones de estudiantes se centraron en indagarlas durante la resolución de problemas matemáticos (ver, por ejemplo, MIDDLETON; JANSEN; GOLDIN, 2018), con el paso del tiempo se ha extendido a estudiarlas durante el proceso de enseñanza y aprendizaje de las matemáticas.

Respecto al bachillerato, en Reino Unido, Lewis (2013) estudió el fenómeno de la 
desafección (motivación y emoción negativas) y encontró que los estudiantes presentan un estrés fuerte hacia las matemáticas como materia escolar, además de ansiedad, humillación, vergüenza y culpa. En España, Pérez-Tyteca, Monje y Castro (2013) encontraron que el examen es la situación que más ansiedad provoca en los estudiantes.

En México, las investigaciones de Martínez-Sierra y García-González (2014; 2017) han profundizado en el estudio de las emociones de estudiantes de bachillerato físico-matemático. Esta modalidad prepara al estudiante en seis semestres para cursar carreras afines a la matemática, y en cada semestre se aborda una de las siguientes materias, álgebra, geometría y trigonometría, geometría analítica, cálculo diferencial, cálculo integral, y probabilidad y estadística. La investigación de estos autores se centró en conocer las emociones de estudiantes que habían reprobado (MARTÍNEZ-SIERRA; GARCÍA-GONZÁLEZ, 2014) y que no habían reprobado ningún curso de matemáticas durante su estancia en el bachillerato (MARTÍNEZSIERRA; GARCÍA-GONZÁLEZ, 2017).

Como resultado encontraron que, independientemente de haber reprobado o no, ambos grupos de estudiantes experimentaron el mismo tipo de emociones. Además identificaron las situaciones desencadenantes de las emociones, entre ellas, la propia clase de matemáticas que desencadenaba miedo, aburrimiento y alivio; la resolución de problemas que desencadenaba satisfacción, decepción, reproche y orgullo y el examen, que originaba júbilo y congoja en los estudiantes. A fin de ampliar la comprensión sobre las situaciones que originan emociones de estudiantes de bachillerato mexicanos, se planteó una investigación a manera de replicación de los estudios realizados por Martínez-Sierra y García-González (2014; 2017, en adelante estudio original). A manera de hipótesis, consideramos que la modalidad del bachillerato influye las emociones de los estudiantes, ya que en un bachillerato físico-matemático hay más asignaturas de matemáticas que en el bachillerato general.

El estudio de replicación (SÁNCHEZ, 2020) se basa en una replicación cerrada, debido a que conservamos el marco teórico-metodológico para la recolección e interpretación de datos, y es de tipo interna ya que la primera autora de este escrito condujo el estudio original. Respecto a la relevancia de estos estudios, se dice que ayudan a probar la veracidad de los resultados del estudio original, más allá del contexto en el que se desarrolló, y para hacerlo existen dos formas, scaling up, que implica utilizar una población de estudio con características similares al estudio original, pero más grande, y scaling out, que conlleva replicar un estudio, pero usar una población de estudio con características distintas a la original (SÁNCHEZ, 2020). Por nuestra parte, nos ceñimos a la forma scaling out, al variar la modalidad del bachillerato. Consideramos que esta relevancia es parte de la originalidad de la investigación aquí descrita. 
Tener conocimiento de las emociones de los estudiantes es importante, ya que se sabe que éstas guían las acciones que los estudiantes realizan en el aula de clases. En el caso de México, se sabe poco de las emociones de los estudiantes en la clase de matemáticas, en general, y del bachillerato, en particular, a diferencia de España, en dónde ya se atiende la regulación emocional en estudiantes de secundaria (ver, por ejemplo, QUINTANILLA; GALLARDO, 2020; CABALLERO, 2020). Un estudio como el que aquí se expone nos acerca a esta habilidad emocional, además consideramos pertinente un estudio de las emociones en bachillerato, porque en este nivel escolar es dónde el estudiante tiene que elegir entre terminar sus estudios o continuar con ellos en la universidad, las emociones que experimente en las clases de matemáticas pueden influir la elección de su futuro académico y laboral.

\section{Las emociones}

\subsection{Emociones académicas}

En el contexto escolar las emociones han ocupado gran atención por parte de investigadores educativos y psicólogos (SCHUTZ; PEKRUN, 2007), producto de las indagaciones se ha denominado emociones académicas a aquellas que se experimentan en el contexto escolar (PEKRUN, 2006). Entre ellas se encuentran tres grupos: 1) emociones de logro, que se relacionan con el éxito o el fracaso de una tarea escolar, como el disfrute y el aburrimiento; 2) emociones sociales, que se manifiestan hacia los demás, por ejemplo, la envidia y la admiración; 3) emociones epistémicas, que tienen como objeto los aspectos del aprendizaje que generan conocimiento como resultado de las cualidades cognitivas y epistémicas de la información y el procesamiento de esa información, entre ellas, la sorpresa, la curiosidad, la confusión, la frustración y la ansiedad (CHEVRIER et al., 2019). Bajo esta perspectiva, podemos decir que el interés de la presente investigación son las emociones académicas, específicamente en matemáticas.

\subsection{Emociones en matemáticas}

En la actualidad se reconoce un vínculo indisociable entre emoción y cognición, y se aconseja estudiar los procesos de enseñanza y aprendizaje de las matemáticas haciendo intervenir las emociones en ellos (QUINTANILLA; GALLARDO, 2020). Sin embargo, este resultado ha sido producto de un gran número de investigaciones a lo largo del tiempo, 
enseguida mostramos algunos resultados relevantes.

Como punto de partida, se sabe que las emociones afectan el proceso cognitivo del estudiante de varias maneras, por ejemplo sesgan su atención y su memoria y activan su tendencia a la acción (HANNULA, 2002; DE BELLIS; GOLDIN, 2006). Particularmente, los estudios de Gómez-Chacón (2000a, 2000b) probaron que la ansiedad y la frustración de los estudiantes están desencadenadas por la dificultad en la comprensión de un problema o en la recuperación de la información de la memoria. Mientras que las emociones positivas, como la alegría y la satisfacción, se desencadenan por la toma de conciencia del progreso personal en el aprendizaje matemático. Sin embargo, también se advierte que no se puede asegurar, de manera absoluta, que todas las emociones positivas facilitan el aprendizaje ni que todas las negativas lo dificulten (GÓMEZ-CHACÓN, 2000b). Más bien, la repercusión y el efecto de las emociones parecen depender de la intensidad de las respuestas emocionales y la interpretación subjetiva que realiza la persona sobre las mismas. De ahí la importancia de los docentes para ayudar a los alumnos a regular las emociones.

El papel del docente en la regulación emocional también fue resaltado por autores como Op’t Eynde, De Corte y Verschaffel (2006) quienes recomiendan que, al enseñar la resolución de problemas matemáticos, se deje espacio para las emociones negativas, ya que solo cuando los estudiantes las experimenten tendrán la oportunidad de aprender a lidiar con ellas. En la actualidad, el interés por el desarrollo emocional de estudiantes ha cobrado fuerza por investigadores y docentes, debido a las nuevas políticas educativas y los avances de la neurociencia.

En el caso particular de México, el modelo educativo vigente de educación básica tiene como un eje rector las habilidades socioemocionales (SEP, 2017). En el caso de la Educación Matemática, cada vez más investigadores centran su atención en las experiencias emocionales de los estudiantes durante la actividad matemática, con la finalidad de mejorar el aprendizaje. A manera de ejemplo, se puede consultar el monográfico de la revista UNO del número 88 (abril-junio 2020) dedicado a las emociones en matemáticas.

\subsection{La Teoría de la estructura cognitiva de las emociones}

Las emociones son objetos de estudio de la Psicología. Desde esta disciplina, existen tres clases de evidencia a las que uno puede recurrir para conocer las emociones de las personas. En primer lugar, se encuentra el lenguaje, la segunda fuente es la observación de la conducta y la tercera fuente es la fisiológica. Nosotros nos adherimos a la fuente del lenguaje, en particular 
desde la teoría de la estructura cognitiva de las emociones (ORTONY; CLORE; COLLINS, 1988) llamada comúnmente teoría OCC.

Desde ella, las emociones son entendidas como "reacciones con valencia ante acontecimientos, agentes u objetos, la naturaleza particular de las cuales viene determinada por la manera como es interpretada la situación desencadenante" (ORTONY; CLORE; COLLINS, 1996, p.16), lo que significa que las emociones son desencadenadas por las valoraciones cognitivas que la gente hace de una situación, de manera consciente o no. La OCC toma como fuente de evidencia de la emoción al lenguaje, a través de él es posible conocer los orígenes y la emoción misma experimentada por las personas, y señala una tipología como la que se muestra en el Cuadro 1.

\begin{tabular}{|c|l|}
\hline Tipo & \multicolumn{1}{|c|}{ Definición } \\
\hline Feliz-por & Contento por un acontecimiento deseable para alguna otra persona \\
Alegre por el mal ajeno & Contento por un acontecimiento indeseable para alguna otra persona \\
Resentido-por & Descontento por un acontecimiento deseable para alguna otra persona \\
Quejoso-por & Descontento por un acontecimiento indeseable para alguna otra persona \\
Esperanza & Contento por la previsión de un acontecimiento deseable \\
Satisfacción & Contento por la confirmación de la previsión de un acontecimiento deseable \\
Alivio & Contento por la refutación de la previsión de un acontecimiento indeseable \\
Decepción & Descontento por la refutación de la previsión de un acontecimiento deseable \\
Miedo & Descontento por la previsión de un acontecimiento indeseable \\
Temores confirmados & Descontento por la confirmación de la previsión de un acontecimiento indeseable \\
Júbilo & Contento por un acontecimiento deseable \\
Congoja & Descontento por un acontecimiento indeseable \\
Orgullo & Aprobación de una acción plausible de uno mismo \\
Aprecio & Aprobación de una acción plausible de otro \\
Autorreproche & Desaprobación de una acción censurable de uno mismo \\
Reproche & Desaprobación de una acción censurable de otro \\
Agrado & Agrado por un objeto atractivo \\
Desagrado & Desagrado por un objeto atractivo \\
\hline
\end{tabular}

Cuadro 1 - Tipología de emociones desde la OCC

Fuente: basado en Ortony, Clore y Collins (1996)

La teoría OCC, al igual que en el estudio original, funge en esta investigación como marco teórico-metodológico. Como sustento teórico nos permitió posicionarnos en una definición precisa de las emociones, a partir de la valoración cognitiva que realiza el estudiante que experimenta la emoción. Como marco metodológico, la tipología de emociones nos señaló la forma de analizar los datos con base en las definiciones de los tipos de emociones.

\section{Metodología}

Al igual que en el estudio original, se adoptó el paradigma cualitativo para dar cuenta de las situaciones que desencadenan las emociones de los estudiantes, ya que la voz de los participantes dicta las situaciones que desencadenan las emociones que experimentan en la clase 
de matemáticas.

\subsection{Participantes y contexto}

A diferencia del estudio original, los participantes fueron estudiantes de un bachillerato general. El bachillerato general tiene una duración de seis semestres, y en ellos se cursan cinco materias de matemáticas, una por semestre: (1) Álgebra, (2) Estadística, (3) Trigonometría, (4) Geometría analítica (5) Probabilidad. Participaron 33 estudiantes (con edades entre 16 y 17 años de edad) de un bachillerato general ubicado en la Ciudad de Chilpancingo de los Bravo, Guerrero, México. Al momento de la investigación estaban cursando Matemáticas IV, correspondiente a Geometría Analítica, dónde se abordan los temas de distancia entre dos puntos, la fórmula de la recta, la circunferencia, la parábola y la elipse. De los 33 estudiantes, solo dieciocho habían reprobado en semestres anteriores, cinco de ellos matemáticas, y trece habían reprobado otras asignaturas, entre ellas química, historia, psicología, artísticas, filosofía y física.

\subsection{Instrumento de recolección de datos}

Como instrumento de recolección de datos se utilizó un cuestionario de preguntas abiertas. Race (2008) señala que un cuestionario está limitado por la cantidad de preguntas que se pueden formular, y puede no proporcionar al investigador la cantidad de detalles relevantes que se pueden obtener a través de métodos cualitativos, como la observación participante, en nuestro caso este señalamiento fue superado, debido a que las preguntas utilizadas en el cuestionario ya habían sido usadas en el estudio original y probada su utilidad para arrojar información sobre las emociones y las situaciones desencadenantes.

El cuestionario se conformó de cuatro preguntas abiertas, las mismas del estudio original. Las preguntas fueron: 1) A lo largo de tu vida académica, ¿qué emociones has experimentado en las clases de matemáticas?, ¿por qué experimentas todo eso? 2) Cuéntame una experiencia positiva que hayas vivido con las matemáticas aquí en el bachillerato, ¿por qué fueron experiencias positivas? 3) Cuéntame una experiencia negativa que hayas vivido con las matemáticas aquí en el bachillerato, ¿por qué fueron experiencias negativas? y 4) Menciona emociones que hallas experimentado en tus clases de matemáticas aquí en el bachillerato. La pregunta 1 pretendía conocer las emociones y situaciones desencadenantes del estudiante durante toda su trayectoria escolar, las preguntas 2 y 3 pretendían conocer solo las emociones 
positivas y negativas, y sus situaciones desencadenantes, en el bachillerato. La pregunta 4, se realizó con el fin de encontrar alguna otra emoción que no fue mencionada en 2 y 3, así como sus respectivas situaciones desencadenantes.

La aplicación del cuestionario se llevó acabo en 2018 y estuvo a cargo de la segunda autora. Para responder el cuestionario se formaron dos grupos, G1 (diecinueve estudiantes) y G2 (catorce estudiantes). Con la finalidad de que todos entendieran las preguntas y las respondieran, al mismo tiempo y de manera completa, se proyectaron en la pizarra y los estudiantes anotaron sus respuestas en hojas que se les entregaron, esto se realizó en un periodo de una hora. Para la identificación de los alumnos se utilizó el código $G_{n}-H_{k} \circ G_{n}-M_{k}$ donde $G$ se refiere al número del grupo, $\mathrm{H}$ y $\mathrm{M}$ se refieren al sexo del estudiante, $\mathrm{n}$ (del 1 al 2) y k (del 1 al 19), es el número asignado al participante.

\subsection{Análisis de Datos}

Al igual que en el estudio original, el análisis de datos se enfocó en codificar la evidencia centrando la atención en las palabras emocionales y frases concisas que permitieran identificar las situaciones desencadenantes de la emoción. Después, las palabras emocionales y las situaciones desencadenantes fueron asociadas a alguno de los tipos de emociones OCC. El análisis de los datos fue realizado en conjunto por las dos primeras autoras, y, para aumentar la validez y confiabilidad de los hallazgos, se compartió con la tercera autora para llevar a cabo una triangulación por investigadores (ROTHBAUER, 2008). A manera de ejemplo, presentamos la siguiente evidencia que da cuenta de una emoción de tipo orgullo.

Una vez nuestro profesor nos dejó unos ejercicios de los cuales resolví 3, me sentí bien [palabra emocional] porque los hice yo, sin ayuda de nadie... fue una experiencia positiva porque lo hice por mí misma [situación desencadenante] (Respuesta de la estudiante G1-M4, 2018).

Debido a que la valoración de la estudiante es en términos de lo que fue capaz de realizar por sí misma, esta evidencia da cuenta de una emoción de tipo orgullo, que se define como aprobación de una acción plausible de uno mismo, la acción plausible de la estudiante es haber realizado los ejercicios, y la aprobación se resalta en la frase me sentí bien. Aclaramos al lector que la teoría OCC señala que la clasificación de emociones no se hace solo por la palabra emocional del que narra la experiencia emocional, sino que, además, se considera la situación que desencadena dicha emoción para comprender porqué se desencadena. 


\section{Resultados}

El análisis de datos arrojó siete situaciones desencadenantes y ocho tipos de emociones de la teoría OCC. Las situaciones que desencadenan las emociones de los estudiantes de bachillerato general son: 1) entender los temas; 2) las preguntas y tareas; 3) acreditar la materia; 4) resolver problemas matemáticos; 5) comprender los temas; 6) el interés por las matemáticas y 7) la dificultad de las matemáticas. Cuando estas situaciones se corresponden con los deseos de los alumnos, entonces las emociones que se desencadenan son positivas; en el caso contrario, se desencadenan las negativas. Para brindar al lector una proyección visual de los hallazgos, la Figura 1 presenta las emociones encontradas en la evidencia. Aclaramos que los datos representan el número de veces que se identificó la emoción, ya que algunos alumnos informaron más de una emoción.

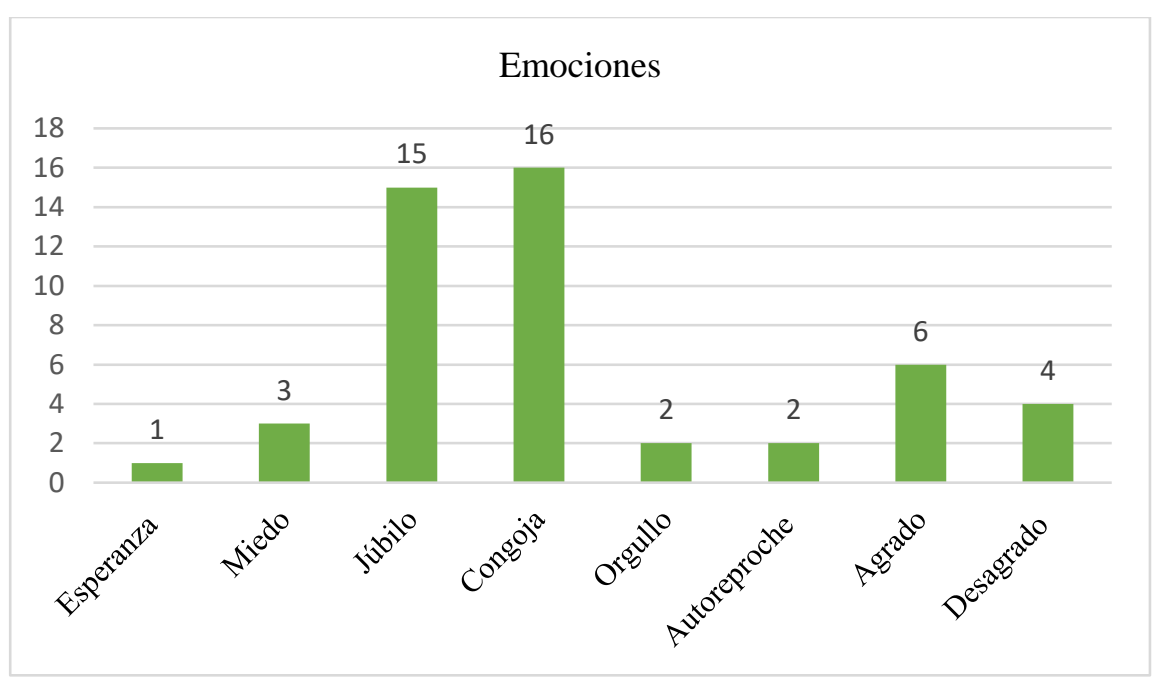

Figura 1 - Emociones de estudiantes de bachillerato en la clase de matemáticas Fuente: elaboración propia

Enseguida se describe cada una de las situaciones desencadenantes y las emociones respectivas. Aclaramos al lector que el reporte sigue la estructura del estudio original, debido a la familiaridad de la primera autora con este tipo de presentación.

\subsection{Situación 1: Entender los temas}

El primer acontecimiento de la clase de matemáticas que identificamos como detonante de las emociones de los estudiantes, es entender los temas, esto hace referencia a entender la explicación del profesor del tema abordado en la clase. Además, si los estudiantes entienden los temas creen que serán capaces de realizar los ejercicios posteriores a la explicación del tema, o de adquirir seguridad para resolver problemas en la pizarra. Por la situación anterior, cuando se 
logra entender los temas se desencadenan emociones positivas como la esperanza y el júbilo, de lo contrario, se desencadenan emociones como la congoja y el desagrado. Enseguida se muestra evidencia de ellas.

\subsubsection{Esperanza}

La esperanza es una emoción basada en previsiones, esto significa que se experimenta cuando se espera que se cumpla determinado acontecimiento. Desde la OCC, la esperanza se define como contento por la previsión de un acontecimiento deseable, en el caso de los estudiantes, interpretamos que cuando entienden los temas prevén que podrán resolver problemas, lo que los hace experimentar un contento que llamamos esperanza. A manera de ejemplo, exponemos el caso de G2-M5, ella señala que cuando entiende los temas se siente contenta, usa la frase me emociono, porque prevé que podrá realizar las actividades que su profesora le encomiende. Además, en su discurso se deja ver la creencia de que entiende el tema porqué su maestra lo explica bien.

Cuando la maestra explica bien el tema sí llego a entender [situación desencadenante], y cuando veo que ya le entiendo pues me emociono [palabra emocional] porque le entendi [situación desencadenante] ... y si la maestra nos deja algún trabajo sobre lo que explicó sé que lo voy a hacer bien [previsión] (Respuesta de la estudiante G2-M5, 2018).

\subsubsection{Júbilo}

El júbilo se desencadena cuando se cumple un acontecimiento que es deseado para la persona, nótese la distinción con la esperanza, en el sentido que ella ocurre cuando el acontecimiento es previsto y el júbilo cuando el acontecimiento se cumple. En el caso de los estudiantes identificamos que entender los temas es un acontecimiento que les genera contento cuando es alcanzado, este contento lo expresan con las palabras emocionales: alegría, emoción, contento, y experiencia positiva. Recordamos al lector que, de acuerdo a los preceptos de la OCC, las palabras emocionales por sí solas no etiquetan los tipos de emociones, sino más bien el uso de ellas junto a la situación desencadenante. En el caso de G1-H8, recuerda entender los temas como un acontecimiento deseable, ya que no todos los estudiantes se interesan por las matemáticas, al parecer él forma parte de los interesados.

Aqui en el bachillerato he vivido bien las matemáticas, porque en algunas clases entiendo los temas [situación desencadenante] y eso me alegra mucho [palabra emocional], fueron experiencias bonitas [palabra emocional], porque no todo el salón le pone interés a las clases de matemáticas (Respuesta del estudiante G1-H8, 2018). 
Encontramos, también, que las emociones de júbilo tienen consecuencias positivas para los estudiantes. Por ejemplo, el estudiante G1-H2 es capaz de resolver problemas, dado que entiende los temas previamente.

A veces siento alegría [palabra emocional] al poder entender los temas que vemos en clases [situación desencadenante] y se me facilita poder resolver problemas de matemáticas [consecuencia] (Respuesta del estudiante G1-H2, 2018).

En el caso de G1-H4, entender los temas de matemáticas, le brindaba seguridad para poder resolver problemas al salir a la pizarra.

He experimentado la emoción de estar contento [palabra emocional] porque la maestra nos explicaba bien y entendía el tema [situación desencadenante], y cuando salía a la pizarra no sentía nervios porque tenía todo entendido [seguridad] (Respuesta del estudiante G1-H4, 2018).

Algo similar le ocurre a la estudiante G2-M1, a quien la seguridad que le da entender los temas de matemáticas, la hace querer participar en clases.

La experiencia positiva [palabra emocional] que he vivido con las matemáticas es cuando entiendo muy bien los temas [situación desencadenante] y me gusta participar en clases y dar puntos de vista de lo que yo entendí [seguridad] (Respuesta de la estudiante G2-M1, 2018).

\subsubsection{Congoja}

La congoja ocurre cuando se cumple un acontecimiento que no se desea. Interpretamos que entender los temas es un acontecimiento deseable, y al contario, no entender los temas es un acontecimiento indeseable. Cuando los estudiantes no logran entender los temas de matemáticas se manifiesta un descontento que expresan con las palabras emocionales, siento nervios, me desespero, siento confusión y enojo.

G1-H2: Siento nervios [palabra emocional], porque hay veces que a los temas de la clase no les entiendo [situación desencadenante] y me desespero [palabra emocional].

G2-H3: La mayoría de las veces siento confusión [palabra emocional], porque algunos temas no los entiendo [situación desencadenante].

G2-M7: En ocasiones me desespero [palabra emocional] porque no entiendo los temas [situación desencadenante] (Respuestas de los estudiantes, 2018).

En el caso de G1-M7 la congoja ocasionada por no entender el tema matemático le impide poder resolver problemas.

Siento enojo [palabra emocional] porque hay veces que no puedo hacer algún ejercicio[consecuencia] por no entender algún tema [situación desencadenante] (Respuesta de la estudiante G1-M7, 2018).

\subsubsection{Júbilo y congoja}

Hubo casos en los que los estudiantes resaltaron dos emociones al mismo tiempo en su 
discurso, se puede apreciar que los estudiantes experimentan júbilo si logran entender los temas, de lo contrario experimentan congoja. Nótese el grado de intensidad de las palabras emocionales que utilizan para expresar la congoja, entre ellas, incomodidad, tristeza, y desesperación.

G2-M2: He experimentado presión, incomodidad [palabras emocionales intensas] porque hay algunos temas que no entiendo[situación desencadenante] ... y alegría [palabra emocional] porque hay temas que entiendo [situación desencadenante].

G1-M7: He sentido alegría o a veces tristeza [palabras emocionales de valencia distinta], alegría por entender los temas [situación desencadenante de la alegria] y tristeza por no entenderlos [situación desencadenante de la tristeza].

G2-M1: Me gustan las matemáticas y me siento contenta [palabras emocionales] cuando logro entender los temas, cuando no logro entenderlos [situaciones desencadenantes] me siento desesperada [palabra emocional intensa] (Respuesta de los estudiantes, 2018).

\subsubsection{Congoja y agrado}

En el caso del estudiante G1-H8 identificamos dos emociones opuestas, el agrado y la congoja, él muestra interés por las matemáticas, lo que da cuenta de una atracción hacia ellas, desde el punto de vista de la OCC, y se corresponde con la definición del agrado, pero asegura que cuando no entiende los temas se enoja, lo que da cuenta de la congoja.

Las emociones que he experimentado son la alegría y el enojo[palabras emocionales de valencia distinta]. Experimento todo eso porque a la vez si me interesan las matemáticas [situación desencadenante], pero me enojo[palabra emocional] cuando no les entiendo a los temas [situación desencadenante] (Respuesta del estudiante G1-H8, 2018).

\subsubsection{Desagrado}

Los casos de G2-M4 y G2-H1, expresan desagrado por las matemáticas, pero detrás de este desagrado se encuentra el acontecimiento entender los temas. En el caso de G2-M4, las matemáticas le desagradan y esta predisposición al disgusto por ellas ocasiona que no entienda los temas. En el caso de G2-H1, no entender los temas debido a la explicación del profesor ocasiona que le disgusten las matemáticas.

G2-M4: A mí las matemáticas [situación desencadenante] no me gustan [palabra emocional], y cuando me explican no entiendo nada [consecuencia].

G2-H1: No me gustan [palabra emocional] las matemáticas porque algunos maestros no explican bien [situación desencadenante] y eso hace que no les entienda a los temas [consecuencia] y no me gusten [palabra emocional] (Respuesta de los estudiantes, 2018).

\subsection{Situación 2: Tareas y preguntas}

Encontramos que las tareas encomendadas por el docente y las preguntas que realiza en 
clase son acontecimientos que desencadenan la emoción de miedo. Si bien la evidencia no deja verlo, consideramos que el miedo hacia estas situaciones puede tener de raíz la comprensión de los temas. Enseguida presentamos la evidencia del miedo.

\subsubsection{Miedo}

El miedo tiene su origen en situaciones que puedan suceder. Cuando los estudiantes no están seguros de sus conocimientos sienten que no serán capaces de responder preguntas en clase (G2-H2), o de realizar las tareas que el profesor les encomiende (G2-M5). En el caso de G2-M5, la evidencia permite ver que manifiesta una predisposición negativa hacia las matemáticas, sin embargo tiene la creencia de que debe aprenderlas.

G2-H2: Siento algo de preocupación [palabra emocional] en la clase de matemáticas porque tal vez vendrá algo que no pueda responder [previsión], por ejemplo alguna pregunta de la clase [situación desencadenante].

G2-M5: A mí eso de las matemáticas no se me da [palabra emocional], pero es necesario aprender matemáticas [creencia] ... siento que si hago alguna tarea que nos deje la maestra la voy a hacer mal [previsión-situación desencadenante] (Respuesta de los estudiantes, 2018).

\subsection{Situación 3: Acreditar la materia}

Acreditar la materia es la tercera situación que identificamos como desencadenante de emociones, en particular el miedo y el júbilo, miedo cuando no se acredita y júbilo cuando logra acreditarse. Consideramos que este hecho obedece a que si el alumno acredita matemáticas, entonces será más probable que se gradúe del bachillerato.

\subsubsection{Miedo y júbilo}

En la respuesta de G1-H5, interpretamos la palabra emoción asociada a un contento, ya que se relaciona con el acontecimiento deseable acreditar la materia, de ahí que la evidencia de este alumno se corresponda con la emoción de júbilo. Por el contrario, cuando este alumno no acredita la materia, experimenta tensión, misma que interpretamos como un descontento, que se corresponde con la definición del miedo.

He experimentado emoción [palabra emocional] al acreditar matemáticas [situación desencadenante], y en ocasiones tensión [palabra emocional] porque he sentido que no voy a pasar la materia [previsión-situación desencadenante] (Respuesta del estudiante G1-H5, 2018). 


\subsection{Situación 4: Resolver problemas}

Esta situación aparece como desencadenante de dos emociones positivas, el júbilo y el orgullo, y dos emociones negativas, la congoja y el autorreproche, todas ellas desencadenadas dependiendo de si se logra, o no, resolver el problema. De acuerdo con la OCC, resolver problemas puede ser interpretado como un acontecimiento de la clase que desencadena emociones de bienestar como el júbilo y la congoja, por su parte, las emociones de orgullo y autorreproche pertenecen al grupo de reacciones ante los agentes, lo que significa que los estudiantes son los autores que desencadenan estas emociones al atribuirse la responsabilidad de resolver, o no, un problema. Enseguida se muestra evidencia de los tipos de emociones identificados.

\subsubsection{Júbilo}

Interpretamos que resolver problemas en clase es un acontecimiento deseable para los estudiantes, y cuando se alcanza, manifiestan un contento que expresan con palabras emocionales como felicidad (G1-H9 y G1-M3) y experiencia positiva (G2-M8), lo que da cuenta de la emoción júbilo.

G1-H9: Mi emoción es de felicidad [palabra emocional] al resolver un problema de matemáticas [situación desencadenante].

G1-M3: Cuando me ponían a resolver problemas y los podía hacer [situación desencadenante] me sentía feliz [palabra emocional].

G2-M8: Mi experiencia positiva [palabra emocional] fue que yo era la que terminaba más pronto los ejercicios que nos dejaba la maestra y mi trabajo estaba muy bien [situación desencadenante] (Respuestas de los estudiantes, 2018).

\subsubsection{Congoja}

Los estudiantes manifiestan un descontento por no alcanzar el acontecimiento deseable resolver problemas, lo que les origina la congoja. En el caso de G2-M3 y G1-M4, expresan el descontento con las palabras emocionales me desespera, me sentía muy mal, sentía tristeza y enojo.

G2-M3: Pues hay veces que las clases son dinámicas ...pero a veces me desespera [palabra emocional] no tener una respuesta a un problema de matemáticas [situación desencadenante]. G1-M4: Me sentía muy mal, sentía tristeza y enojo [palabras emocionales] cuando mis respuestas de los problemas eran incorrectas [situación desencadenante] (Respuestas de los estudiantes, 2018). 


\subsubsection{Orgullo}

Interpretamos que resolver problemas en la clase de matemáticas es un acontecimiento elogiable por parte del profesor y de los estudiantes, así, quienes logran resolver problemas experimentan emociones de orgullo. Desde la OCC, si el estudiante aprueba, así mismo, la acción de poder resolver problemas, manifiesta orgullo. En la evidencia encontramos esta aprobación expresada en las frases, me superé a mi mismo (G1-H11), lo hice por mí misma (G1M4), у $\operatorname{orgullo}(\mathrm{G} 2-\mathrm{H} 3)$.

G1-H11: Una experiencia positiva [palabra emocional] fue cuando la maestra nos puso un problema y lo resolví sin ayuda de mis compañeros [situación desencadenante] y me superé a mi mismo [palabra emocional].

G1-M4: Una vez nuestro profesor nos dejó unos problemas y los resolvi [situación desencadenante], me senti bien porque los hice yo, sin ayuda de nadie... fue una experiencia positiva porque lo hice por mí misma [palabras emocionales].

G2-H3: He experimentado orgullo [palabra emocional] al poder resolver problemas que antes no podía [situación desencadenante] (Respuestas de los estudiantes, 2018).

\subsubsection{Autorreproche}

Los estudiantes expresaron esta emoción al no lograr resolver problemas. Ellos evidencian la desaprobación de este acontecimiento con las frases me sentí mal de no superarme a mí mismo (G1-H11) y sentí un poco de enojo conmigo mismo (G1-H10). En el caso de G1H11 el no resolver problemas le trajo como consecuencia negativa no tener participación en clase, la importancia que este alumno le da a las participaciones es porque éstas forman parte de la evaluación de la asignatura de matemáticas.

G1-H11: Una experiencia negativa [palabra emocional] fue cuando la maestra nos puso un problema, nos dio un tiempo para resolver y no pude resolverlo, no me puso participación [situaciones desencadenantes], me molesté y me sentí mal [palabra emocional].

G1-H10: En una clase estaba resolviendo ecuaciones y no pude resolver algunas, sentí un poco de enojo conmigo mismo [palabra emocional] por no poder resolverlas [situación desencadenante] (Respuestas de los estudiantes, 2018).

\subsection{Situación 5: Comprender los temas}

La quinta situación que identificamos la hemos denominado comprender los temas, hacemos la distinción con la situación 1, entender los temas, en el sentido que ésta se refiere a entender la explicación de los temas, y la situación 5 hace referencia a poder aplicar lo aprendido. Identificamos dos emociones desencadenadas por comprender los temas, la congoja y el agrado. Desde la OCC, la congoja es una emoción de bienestar, lo que indica que se 
desencadena ante la deseabilidad del acontecimiento comprender los temas, por su parte el agrado es una emoción de atracción, por ello interpretamos comprender los temas como un objeto agradable para el estudiante. Enseguida detallamos evidencia de estas emociones.

\subsubsection{Congoja}

Interpretamos la comprensión de los temas como un acontecimiento deseable para los estudiantes, ya que si comprenden los temas será más probable resolver problemas o acreditar exámenes. En el caso de los estudiantes G2-M1 y G1-H4 manifiestan congoja al no poder fundamentar el resultado de una operación y no poder resolver un problema en clase o en el examen. Por nuestra parte, consideramos que puede ser debido a que los temas no han quedado comprendidos. Los estudiantes manifiestan el descontento con las frases experiencia negativa y me he sentido nervioso.

G2-M1: La experiencia negativa [palabra emocional] que he vivido en el bachillerato ha sido el examen, cuando me quedo en alguna ecuación y no recuerdo el procedimiento y no puedo resolverla [situación desencadenante].

G1-H4: Me he sentido nervioso [palabra emocional] cuando me pasan a resolver problemas a la pizarra [situación desencadenante] (Respuestas de los estudiantes, 2018).

\subsection{Situación 6: Interés por las matemáticas}

\subsubsection{Agrado}

El agrado es una emoción de tipo atracción, lo que significa que el sujeto valora la situación desencadenante como un objeto, en este caso, las matemáticas. En los testimonios de los estudiantes asociamos las palabras alegría (G1-H10) y emoción (G1-M5) como evidencia de la atracción hacia las matemáticas, esta atracción la hemos codificado como interés por las matemáticas.

G1-H10: Pues la emoción que he tenido es alegría [palabra emocional] porque me dan ganas de aprender un poco más sobre las matemáticas [situación desencadenante].

G1-M5: He sentido emoción [palabra emocional] por saber más matemáticas [situación desencadenante] (Respuestas de los estudiantes, 2018).

\subsection{Situación 7: Dificultad de las matemáticas}

Los estudiantes manifiestan rechazo hacia las matemáticas, por la dificultad de ésta. Este rechazo da cuenta de la emoción de atracción llamada desagrado, enseguida se muestra 
evidencia de ello.

\subsubsection{Desagrado}

En los casos de G2-H1 y G2-M5, encontramos que valoran a las matemáticas como un objeto repulsivo, y se debe a la dificultad que perciben de ellas. Los estudiantes expresan el rechazo hacia las matemáticas con la palabra emocional no me gustan, sin embargo creen que deben aprenderlas.

G2-H1: Pues no me gustan mucho [palabra emocional] las matemáticas porque se me hacen un poco complicadas [situación desencadenante], pero creo que le debo poner un poco de interés para aprenderlas mejor [creencia].

G2-M5: Pues a mi las matemáticas no me gustan [palabra emocional], pero ahora veo que son importantes [creencia]... hay veces que les entiendo, pero hay veces que no, que se me dificultan [situación desencadenante], pero a pesar de que se me dificulten sé que debo aprenderlas [creencia] (Respuestas de los estudiantes, 2018).

\section{Discusión y conclusiones}

La presente investigación es un estudio de replicación y se planteó como objetivo conocer más sobre las situaciones que originan las emociones de estudiantes de bachillerato en México. La replicación consistió en modificar la población de estudio, considerando el bachillerato general, y se conservó el marco teórico-metodológico y el diseño de la investigación. Con base en los hallazgos podemos decir que la modalidad del bachillerato no influye las emociones de los estudiantes, pues se encontraron emociones y situaciones desencadenantes parecidas al estudio original. La explicación que damos a este hecho es que lo que desencadena las emociones es el cumplimiento de las metas académicas que se plantea el estudiante, si éstas se cumplen las emociones que se experimentan son positivas, de lo contrario son negativas, resultado que coincide con el estudio original.

Las metas académicas que identificamos resultan ser el conjunto de actividades escolares, como las participaciones en clase, resolver ejercicios o problemas, entender los temas, acreditar el examen y graduarse del bachillerato, y éstas trascienden la modalidad del bachillerato, incluso están en otras asignaturas. De esta manera, nuestro estudio confirma que las emociones de los estudiantes de matemáticas no solo dependen de la interpretación subjetiva que realizan los estudiantes de las situaciones que experimentan (GÓMEZ-CHACÓN, 2000a) sino que esta interpretación está en función del cumplimiento de metas académicas personales del estudiante (MARTÍNEZ-SIERRA; GARCÍA-GONZÁLEZ, 2014; 2017). 
Por otro lado, se sabe que las emociones activan la tendencia a la acción de los estudiantes (HANNULA, 2002; DE BELLIS; GOLDIN, 2006). En el caso de nuestros hallazgos, encontramos evidencia de la forma en que actúan los estudiantes cuando experimentan desagrado por las matemáticas, cuando esto ocurre se consideran incapaces de entender los temas, o cuando experimentan miedo se activa su predisposición a creer que en un futuro volverán a fracasar. Pero, también encontramos que cuando experimentan júbilo se sienten seguros para realizar alguna actividad de la clase. Como conclusión de los hallazgos podemos decir que nuestro estudio confirma los resultados del estudio original, las emociones de los estudiantes de bachillerato tienen como situaciones desencadenantes metas académicas, de esta manera el cumplimiento de ellas es lo que provoca emociones positivas o negativas.

Al respecto, una clave hacia la regulación emocional es modificar las situaciones desencadenantes de las emociones negativas y potenciar las de las emociones positivas, por ejemplo, ante el desagrado por las matemáticas se pueden buscar alternativas para revertirlo, desde la inclusión de actividades que sean atractivas para el estudiante hasta mostrarle una matemática que sea importante para él. Enseguida, para profundizar las conclusiones, presentamos la comparación de emociones por modalidad de bachillerato, después hablamos de la relevancia de un estudio de replicación y concluimos con algunas implicaciones del trabajo realizado y con sus limitaciones.

\subsection{Emociones por tipo de bachillerato}

En el Cuadro 2, se exponen las situaciones desencadenantes de las emociones encontradas en el bachillerato físico-matemático y en el general, con la finalidad de mostrar coincidencias y diferencias. Se resaltan, en cursiva, las situaciones desencadenantes similares.

\begin{tabular}{|c|c|c|c|}
\hline \multirow{3}{*}{$\begin{array}{c}\text { Tipo } \\
\text { de emoción }\end{array}$} & \multicolumn{3}{|c|}{ Situación desencadenante } \\
\hline & \multirow{2}{*}{$\begin{array}{l}\text { Bachillerato General } \\
\text { Nuestros resultados }\end{array}$} & \multicolumn{2}{|c|}{$\begin{array}{c}\text { Bachillerato } \\
\text { Estudio original }\end{array}$} \\
\hline & & $\begin{array}{c}\text { Martínez-Sierra y } \\
\text { García-González (2017) }\end{array}$ & $\begin{array}{c}\text { Martínez-Sierra y } \\
\text { García-González (2014) }\end{array}$ \\
\hline Esperanza & $\begin{array}{l}\text { Entender los temas de } \\
\text { matemáticas }\end{array}$ & $\begin{array}{l}\text { No se encontró esta } \\
\text { emoción }\end{array}$ & $\begin{array}{l}\text { No se encontró esta } \\
\text { emoción }\end{array}$ \\
\hline Miedo & $\begin{array}{c}\text { Preguntas y tareas } \\
\text { No acreditar la materia }\end{array}$ & $\begin{array}{l}\text { No entender los temas } \\
\text { Aprender o no en clases } \\
\text { No pasar un examen }\end{array}$ & Clase de matemáticas \\
\hline Júbilo & $\begin{array}{c}\text { Acreditar la materia } \\
\text { Entender los temas de } \\
\text { matemáticas } \\
\text { Resolver problemas }\end{array}$ & $\begin{array}{c}\text { Que la clase termine } \\
\text { Resolver problemas en la } \\
\text { pizarra }\end{array}$ & Presentar un examen \\
\hline Congoja & $\begin{array}{l}\text { No entender los temas } \\
\text { Resolver problemas }\end{array}$ & $\begin{array}{c}\text { No poder resolver un } \\
\text { problema en clase o en el }\end{array}$ & Clase de matemáticas \\
\hline
\end{tabular}




\begin{tabular}{|c|c|c|c|}
\hline & $\begin{array}{c}\text { No comprender los } \\
\text { temas }\end{array}$ & $\begin{array}{c}\text { examen } \\
\text { Pasar al pizarrón }\end{array}$ & \\
\hline Orgullo & Resolver problemas & $\begin{array}{c}\text { Pasar las materias } \\
\text { Resolver un problema }\end{array}$ & $\begin{array}{c}\text { Resolver problemas en el } \\
\text { pizarrón }\end{array}$ \\
\hline Autorreproche & $\begin{array}{c}\text { No poder resolver } \\
\text { problemas }\end{array}$ & $\begin{array}{c}\text { No poder resolver un } \\
\text { problema }\end{array}$ & $\begin{array}{c}\text { No resolver problemas en } \\
\text { el pizarrón }\end{array}$ \\
\hline Agrado & $\begin{array}{c}\text { Interés por las } \\
\text { matemáticas } \\
\text { Entender los temas }\end{array}$ & $\begin{array}{c}\text { Entender las } \\
\text { matemáticas }\end{array}$ & $\begin{array}{c}\text { No enoción } \\
\text { emtá esta }\end{array}$ \\
\hline Desagrado & $\begin{array}{c}\text { Dificultad de las } \\
\text { matemáticas } \\
\text { No entender los temas }\end{array}$ & No poder resolver un \\
& problema & No se encontró esta \\
& emoción
\end{tabular}

Cuadro 2 - Comparación de situaciones desencadenantes en los distintos bachilleratos

Fuente: elaborado por el autor

Respecto al tipo de emociones, nuestros resultados señalan solo uno que no fue reportado por Martínez-Sierra y García-González (2014; 2017): se trata de la emoción de esperanza. Las emociones ya reportadas que encontramos fueron, júbilo, congoja, orgullo, autorreproche, gusto y disgusto, y solo difieren las situaciones que las desencadenan. Como puede notarse en el Cuadro 2, resolver problemas es la situación que, en ambos estudios, desencadenan más emociones de los estudiantes, este resultado lo explicamos debido a que en la clase de matemáticas en general, la actividad de resolver problemas es usada a manera de ejercitación de los conceptos y de evaluación del aprendizaje. Una diferencia que llama la atención del estudio original, es que en el caso de los estudiantes que reprobaron matemáticas la clase de matemáticas en sí misma fue detonante de emociones negativas como la congoja y el miedo, creemos que este resultado se debe a la experiencia misma de reprobación.

En el caso nuestro, no encontramos alguna diferencia relevante entre los cinco estudiantes que habían reprobado matemáticas. Por otro lado, nuestros resultados son coincidentes con los resultados de Lewis (2013), ya que encontramos que los estudiantes manifiestan congoja y autorreproche hacia las matemáticas como materia escolar. PérezTyteca, Monje y Castro (2013) reportan que el examen es la situación que más ansiedad provoca en los estudiantes de bachillerato, por nuestra parte encontramos que entender los temas es la situación que más emociones, tanto positivas como negativas, desencadena.

\subsection{Ventajas del estudio de replicación}

De acuerdo con Sánchez (2020) la literatura especializada muestra varios argumentos sobre la importancia de los estudios de replicación. Entre ellos se dice que en una replicación interna, como la conducida en este estudio, el investigador tiene toda la información necesaria disponible para una replicación fiel del estudio original, además de que está familiarizado con 
los instrumentos, métodos y contribuciones teóricas del estudio original. Esta familiaridad le permite replicar y contribuir al cuerpo de conocimientos sobre un tema. En nuestro caso, la familiaridad de la primera autora con el estudio original permitió que el diseño de investigación fuera lo más apegado al estudio original, salvo que no fue posible el uso de entrevistas como el estudio original lo sugería, pues la institución de los participantes no lo permitió, pero se recurrió al cuestionario de preguntas abiertas, conservando las preguntas del estudio original.

Schmidt (2009, citado en SÁNCHEZ, 2020) afirma que cuando una replicación demuestra que los mismos hallazgos pueden ser obtenidos en cualquier otro lugar por cualquier otro investigador, la replicación se concibe como una operacionalización de la objetividad. En este sentido, consideramos que la relevancia de nuestro estudio estriba en que se comprobó que las emociones de los estudiantes de bachillerato tienen como situaciones desencadenantes las metas académicas tal como lo sugería el estudio original.

\subsection{Emociones académicas y la teoría OCC}

Las emociones que hemos encontrado son propias de la clase de matemáticas y están asociadas a metas que el estudiante desea alcanzar en el salón de clases, por ello se corresponde con la tipología de emociones de logro de las emociones académicas (PEKRUN, 2006), referidas al éxito o al fracaso de una actividad. Por ello, consideramos que la teoría OCC es una herramienta analítica con potencial para reconocer las emociones académicas y las situaciones que las desencadenan. Al respecto, en el Cuadro 3, hemos adaptado las definiciones de la teoría OCC con base en la situación desencadenante que encontramos en nuestro estudio. 


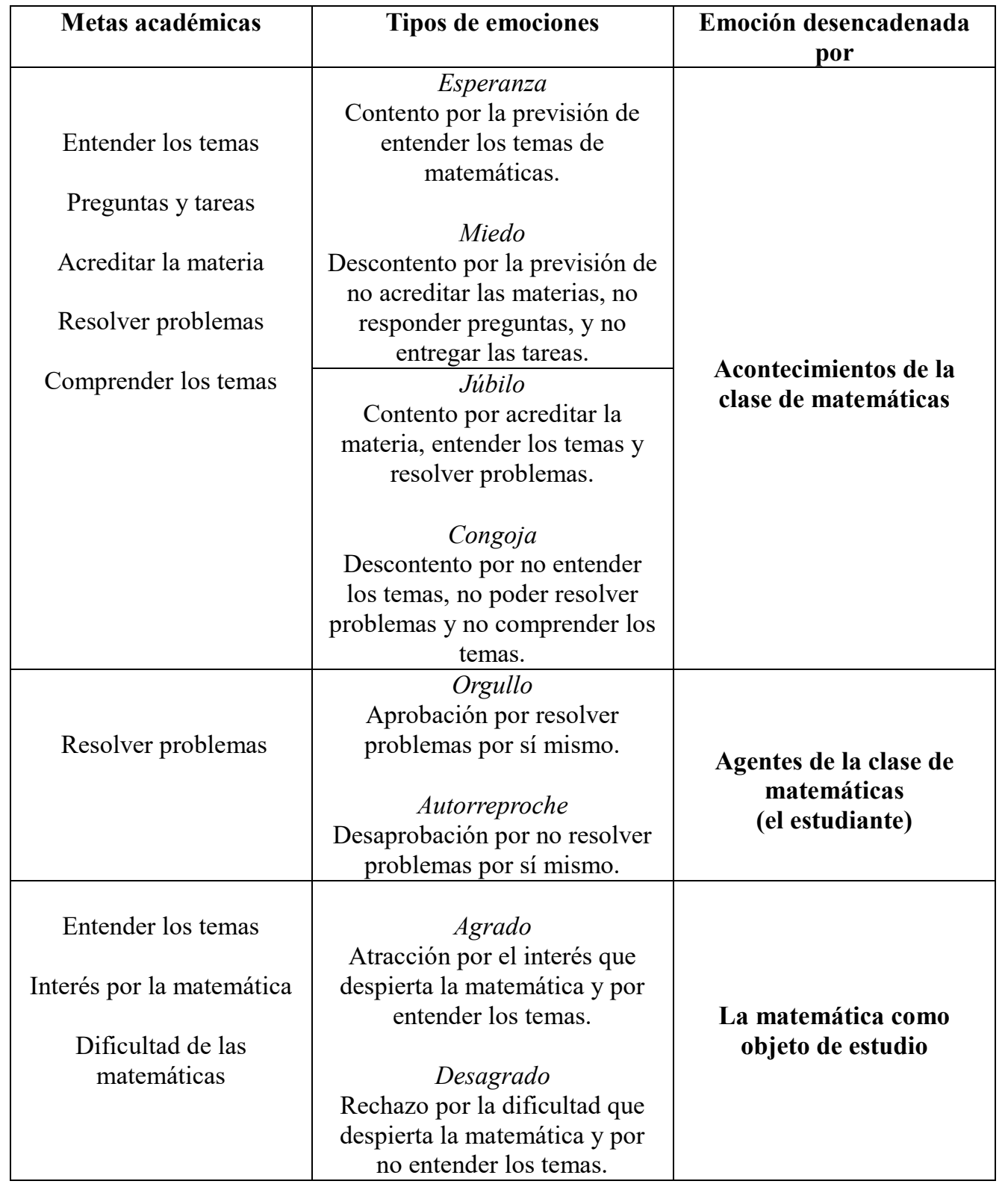

Cuadro 3 - Emociones académicas de logro en matemáticas

Fuente: elaborado por el autor

\subsection{Implicaciones}

Como implicaciones del estudio, señalamos el conocimiento emocional (GARCÍAGONZÁLEZ; MARTÍNEZ-PADRÓN, 2020), referido a la información que se tiene de las emociones que se experimentan. Si los estudiantes son capaces de reconocer la emoción que experimentan y la situación que la desencadena, es más probable que puedan regular sus emociones y lograr un clima idóneo para el aprendizaje de las matemáticas. Al respecto, las preguntas del cuestionario usado en nuestra investigación pueden ser una herramienta muy útil para que los estudiantes expresen las emociones pasadas y presentes de sus clases de matemáticas, y junto con ello desarrollar su conocimiento emocional. 
Además, con el fin de comprender mejor las emociones de estudiantes de matemáticas de bachillerato, se podrían seguir realizando estudios de replicación, pero del estilo replicación conceptual, utilizando métodos de investigación y enfoques teóricos diferentes a los implementados en el estudio original. En correspondencia con lo anterior, señalamos una limitación del estudio original, y se refiere al uso de una sola fuente para la recolección de datos, que pudiera ser complementado con entrevistas individuales u observaciones de clase.

\section{Referencias}

CABALLERO, A. Actividades para el desarrollo de competencias emocionales en el aula de matemáticas. UNO, Madrid, n. 88, p. 34-38, abr. 2020.

CHEVRIER, M. et al. Exploring the antecedents and consequences of epistemic emotions. Learning and instruction, London, v. 63, p. 1-18, 2019.

De BELLIS, V.; GOLDIN, G. Affect and meta-affect in mathematical problem solving: a representational perspective. Educational Studies in Mathematics, Dordrecht, v. 63, n. 2, p. 131-147, 2006.

DI MARTINO, P.; ZAN, R. Attitude towards mathematics: A bridge between beliefs and emotions. ZDM-The international Journal on Mathematics Education, Karlsruhe, v. 43, p. 471-482, 2011.

DI MARTINO, P.; SABENA, C. Elementary pre-service teachers' emotions: shadows from the past to the future. En: KISLENKO, K. (ed.). Current state of research on mathematical beliefs XVI, Tallinn: University, 2011. p. 89-105.

DIEGO-MANTECÓN, J. M.; CÓRDOBA-GÓMEZ, F. J. Adaptación y validación del MRBQ (Mathematics-Related Beliefs Questionnaire) al contexto colombiano con estudiantes de secundaria. Revista Educación Matemática, Ciudad de México, v. 31, n. 1, p. 66-88, 2019.

GARCÍA-GONZÁLEZ, M. S.; MARTÍNEZ-SIERRA, G. Investigación sobre emociones en la clase de matemáticas. En: DOLORES FLORES; C. et al. (eds.). Investigaciones en dominio afectivo en matemática educativa. México: Ediciones Eón. México, 2018. p. 19-38.

GARCÍA-GONZÁLEZ, M. S.; MARTÍNEZ-PADRÓN, O. J. Conocimiento emocional de profesores de matemáticas, Revista Educación Matemática, Ciudad de México, v. 32, n. 1, p. 153-173. 2020.

GÓMEZ-CHACÓN, I. Matemática Emocional. Madrid: Narcea, 2000a.

GÓMEZ- CHACÓN, I. M. Affective influences in the knowledge of mathematics. Educational Studies in Mathematics, Dordrecht, v. 43, n.1, p. 149-168, 2000 b.

HANNULA, M. S. Attitude towards mathematics: emotions, expectations and values. Educational Studies in Mathematics, Dordrecht, v. 49, n. 1, p. 25-46, 2002.

IBARRA-GONZÁLEZ, K. P.; ECCIUS-WELLMANN, C. Desarrollo y validación de un instrumento de medición de la afectividad respecto a la comisión de errores en matemáticas. Bolema, Rio Claro, v. 32, n. 61, p. 673-695, ago. 2018.

LEWIS, G. Emotion and disaffection with school mathematics, Research in Mathematics Education, London, v. 15, n. 1, p. 70-86, 2013. 
MARTÍNEZ-SIERRA, G.; GARCÍA-GONZÁLEZ, G. M. S. Students' Emotions in the High School Mathematical Class: Appraisals in Terms of a Structure of Goals. International Journal of Science and Mathematics Education, Amsterdam, v. 15, n. 2, p. 349-369, 2017.

MARTÍNEZ-SIERRA, G.; GARCÍA-GONZÁLEZ, G. M. S. High school students' emotional experiences in mathematics classes. Research in Mathematics Education, Washington, D.C., v. 16, n. 3, p. 234-250, 2014.

MARTÍNEZ-PADRÓN, O. J. Actitudes hacia la Matemática. Sapiens, Caracas, v. 9, n. 2, p. 237-256, 2008.

MIDDLETON, J.; JANSEN, A.; GOLDIN, G. The Complexities of Mathematical Engagement: Motivation, Affect, and Social Interactions. En: CAI, J. (ed.). COMPENDIUM for Research in Mathematics Education. Virginia: National Council of Teachers of Mathematics, 2018. p. 667-699.

ORTONY, A.; CLORE, G.; COLLINS, A. La estructura cognitiva de las emociones. Ciudad de México: Siglo veintiuno, 1996.

OP 'T EYNDE, P.; DE CORTE, E.; VERSCHAFFEL, L. Accepting emotional complexity: a socioconstructivist perspective on the role of emotions in the mathematics classroom. Educational Studies in Mathematics, Dordrecht, v. 63, n. 2, p. 193-207, 2006.

PEKRUN, R. The control-value theory of achievement emotions: Assumptions, corollaries, and implications for educational research and practice. Educational Psychology Review, Amsterdam, v. 18, n. 4, p. 315-341, 2006.

PÉREZ-TYTECA, P.; MONJE, J.; CASTRO, E. Afecto y matemáticas: Diseño de una entrevista para acceder a los sentimientos de alumnos adolescentes. Avances de Investigación en Educación Matemática, Madrid, v. 4, p. 65-82, 2013.

QUINTANILLA, A.; GALLARDO, J. Identificar experiencias emocionales para mejorar la comprensión en matemáticas. UNO, Madrid, n. 88, p. 24-33, abr. 2020.

RACE, R. Education, qualitative research in. En: GIVEN, L. M. (ed.). The SAGE encyclopedia of qualitative research methods. New York: Sage, 2008. p. 241-244.

RETANA-ALVARADO, D. A.; DE LAS HERAS, M. Á.; VÁZQUEZ-BERNAL, B.; JIMÉNEZPÉREZ, R. ¿Cómo cambian las emociones en docentes en formación inicial al participar en un proyecto de indagación de aula? Ápice, La coruña, v. 3, n. 2, p. 55-69, 2019.

ROTH, W-M.; WALSHAW, M. Affect and emotions in mathematics education: toward a holistic psychology of mathematics. Educational Studies in Mathematics, Dordrecht, v. 102, n. 1, p.111125, 2019.

ROTHBAUER, P. Triangulation. En: GIVEN, L. M. (ed.). The SAGE encyclopedia of qualitative research methods. New York: Sage, 2008. p. 893-894.

SÁNCHEZ, M. Replication Studies in Mathematics Education: What Kind of Questions Would Be Productive to Explore? International Journal of Science and Mathematics Education, Amsterdam, v. 18, n. 1, p. 37-50, 2020.

SCHUTZ, P.; PEKRUN, R. Emotion in education. New York: Academic Press, 2007.

SECRETARIA DE EDUCACIÓN PÚBLICA- SEP. Nuevo modelo educativo. México: SEP, 2017.

SHAO, K.; PEKRUN, R.; NICHOLSON, L. Emotions in classroom language learning: What can we 
learn from achievement emotion research? Learning and instruction, London, v. 86, p. 1-11, 2019.

Submetido em 03 de Abril de 2020. Aprovado em 22 de Outubro de 2020. 